\title{
Editorial \\ Publisher's Note: We Changed Page Numbers to Article Numbers for Articles Published in Clinics and Practice Volumes 1-10
}

\section{Clinics and Practice Editorial Office}

check for updates

Citation: Clinics and Practice Editorial Office. Publisher's Note: We

Changed Page Numbers to Article Numbers for Articles Published in Clinics and Practice Volumes 1-10. Clin. Pract. 2022, 12, 147-156. https://doi.org/10.3390/ clinpract12020019

Received: 22 February 2022 Accepted: 22 February 2022 Published: 23 February 2022

Publisher's Note: MDPI stays neutral with regard to jurisdictional claims in published maps and institutional affiliations.

Copyright: (C) 2022 by the author Licensee MDPI, Basel, Switzerland. This article is an open access article distributed under the terms and conditions of the Creative Commons Attribution (CC BY) license (https:// creativecommons.org/licenses/by/ $4.0 /)$.
MDPI AG, St. Alban-Anlage 66, 4052 Basel, Switzerland; clinpract@mdpi.com

Clinics and Practice [1] was published by PAGEPress from Volume 1 (2011) to Volume 10 (2020). From Volume 11, Issue 1 (2021), Clinics and Practice is published by MDPI.

Previous articles in Volumes 1-10, published by PAGEPress in Open Access under a CC-BY (or CC-BY-NC-ND) licence, are now hosted by MDPI on mdpi.com as a courtesy and upon agreement with PAGEPress.

To standardize the metadata format of all previous articles, MDPI republished 513 articles in Volumes 1-10 with article numbers replacing page numbers (Table 1).

Table 1. MDPI changed page numbers to articles numbers for 513 articles in Volumes 1-10.

\begin{tabular}{|c|c|c|}
\hline DOI & Previous Page Number & Current Article Number \\
\hline 10.4081/cp.2011.e1 & $1-2$ & e1 \\
\hline 10.4081/cp.2011.e2 & 3 & e2 \\
\hline 10.4081/cp.2011.e3 & $4-5$ & e3 \\
\hline 10.4081/cp.2011.e4 & 6 & e4 \\
\hline 10.4081/cp.2011.e5 & $7-8$ & e5 \\
\hline 10.4081/cp.2011.e6 & $9-10$ & e6 \\
\hline 10.4081/cp.2011.e7 & $11-13$ & e7 \\
\hline 10.4081/cp.2011.e8 & 14 & e8 \\
\hline 10.4081/cp.2011.e9 & $15-16$ & e9 \\
\hline 10.4081/cp.2011.e10 & 17 & e10 \\
\hline 10.4081/cp.2011.e11 & $18-19$ & e11 \\
\hline 10.4081/cp.2011.e12 & $20-21$ & e12 \\
\hline 10.4081/cp.2011.e13 & $22-23$ & e13 \\
\hline 10.4081/cp.2011.e14 & 24 & e14 \\
\hline 10.4081/cp.2011.e15 & 25 & e15 \\
\hline 10.4081/cp.2011.e16 & $26-27$ & e16 \\
\hline 10.4081/cp.2011.e17 & $28-29$ & e17 \\
\hline 10.4081/cp.2011.e18 & 30 & e18 \\
\hline 10.4081/cp.2011.e19 & $31-32$ & e19 \\
\hline 10.4081/cp.2011.e20 & $33-34$ & $\mathrm{e} 20$ \\
\hline 10.4081/cp.2011.e21 & $35-36$ & e21 \\
\hline 10.4081/cp.2011.e22 & $37-38$ & $\mathrm{e} 22$ \\
\hline 10.4081/cp.2011.e23 & $39-40$ & e23 \\
\hline 10.4081/cp.2011.e24 & $41-42$ & e24 \\
\hline 10.4081/cp.2011.e25 & $43-45$ & e25 \\
\hline 10.4081/cp.2011.e26 & $46-47$ & e26 \\
\hline 10.4081/cp.2011.e27 & $48-49$ & e27 \\
\hline 10.4081/cp.2011.e28 & $50-51$ & $\mathrm{e} 28$ \\
\hline 10.4081/cp.2011.e29 & $52-53$ & e29 \\
\hline 10.4081/сp.2011.e30 & $54-55$ & e30 \\
\hline 10.4081/cp.2011.e31 & $56-58$ & e31 \\
\hline
\end{tabular}


Table 1. Cont.

\begin{tabular}{|c|c|c|}
\hline DOI & Previous Page Number & Current Article Number \\
\hline 10.4081/cp.2011.e32 & $59-60$ & e32 \\
\hline 10.4081/cp.2011.e33 & $61-62$ & e33 \\
\hline 10.4081/cp.2011.e34 & $63-64$ & e34 \\
\hline 10.4081/cp.2011.e35 & $65-66$ & e35 \\
\hline 10.4081/cp.2011.e36 & $67-69$ & e36 \\
\hline 10.4081/cp.2011.e38 & $72-73$ & e38 \\
\hline 10.4081/cp.2011.e39 & $74-75$ & e39 \\
\hline 10.4081/cp.2011.e40 & 76 & $\mathrm{e} 40$ \\
\hline 10.4081/cp.2011.e41 & $77-78$ & e41 \\
\hline 10.4081/cp.2011.e42 & $79-81$ & $\mathrm{e} 42$ \\
\hline 10.4081/cp.2011.e43 & $82-83$ & $\mathrm{e} 43$ \\
\hline 10.4081/cp.2011.e44 & $84-87$ & e44 \\
\hline 10.4081/cp.2011.e45 & $88-90$ & $\mathrm{e} 45$ \\
\hline 10.4081/cp.2011.e46 & $91-93$ & $\mathrm{e} 46$ \\
\hline 10.4081/cp.2011.e47 & $94-95$ & e47 \\
\hline 10.4081/cp.2011.e48 & $96-97$ & $\mathrm{e} 48$ \\
\hline 10.4081/cp.2011.e49 & 98-99 & e49 \\
\hline 10.4081/cp.2011.e50 & $100-101$ & e50 \\
\hline 10.4081/cp.2011.e51 & $102-104$ & e51 \\
\hline 10.4081/cp.2011.e52 & $105-106$ & e52 \\
\hline 10.4081/cp.2011.e53 & $107-109$ & e53 \\
\hline 10.4081/cp.2011.e54 & 110 & e54 \\
\hline 10.4081/cp.2011.e55 & $111-113$ & e55 \\
\hline 10.4081/cp.2011.e56 & 114-115 & e56 \\
\hline 10.4081/cp.2011.e57 & $116-118$ & e57 \\
\hline 10.4081/cp.2011.e58 & $119-120$ & e58 \\
\hline 10.4081/cp.2011.e59 & $121-122$ & e59 \\
\hline 10.4081/cp.2011.e60 & $123-125$ & e60 \\
\hline 10.4081/cp.2011.e61 & $126-127$ & e61 \\
\hline 10.4081/cp.2011.e62 & $128-129$ & e62 \\
\hline 10.4081/cp.2011.e63 & $130-131$ & e63 \\
\hline 10.4081/cp.2011.e64 & $132-133$ & e64 \\
\hline 10.4081/cp.2011.e65 & $134-135$ & e65 \\
\hline 10.4081/cp.2011.e66 & $136-138$ & e66 \\
\hline 10.4081/cp.2011.e67 & 139-141 & e67 \\
\hline 10.4081/cp.2011.e68 & $142-143$ & e68 \\
\hline 10.4081/cp.2011.e69 & 144 & e69 \\
\hline 10.4081/cp.2011.e70 & $145-146$ & e70 \\
\hline 10.4081/cp.2011.e71 & $147-148$ & e71 \\
\hline 10.4081/cp.2011.e72 & $149-150$ & e72 \\
\hline 10.4081/cp.2011.e73 & $151-152$ & $\mathrm{e} 73$ \\
\hline 10.4081/cp.2011.e74 & $153-154$ & e74 \\
\hline 10.4081/cp.2011.e75 & $155-156$ & e75 \\
\hline 10.4081/cp.2011.e76 & $157-158$ & e76 \\
\hline 10.4081/cp.2011.e77 & $159-161$ & e77 \\
\hline 10.4081/cp.2011.e78 & $162-163$ & $\mathrm{e} 78$ \\
\hline 10.4081/cp.2011.e79 & $164-166$ & e79 \\
\hline 10.4081/cp.2011.e81 & $169-171$ & e81 \\
\hline 10.4081/cp.2011.e82 & $172-173$ & e82 \\
\hline 10.4081/cp.2011.e83 & $174-178$ & e83 \\
\hline 10.4081/cp.2011.e84 & 179 & e84 \\
\hline 10.4081/cp.2011.e85 & 180-181 & e85 \\
\hline 10.4081/cp.2011.e86 & 182-184 & e86 \\
\hline 10.4081/cp.2011.e87 & $185-186$ & e87 \\
\hline 10.4081/cp.2011.e88 & $187-188$ & e88 \\
\hline 10.4081/cp.2011.e89 & 189-192 & e89 \\
\hline 10.4081/cp.2011.e90 & 193-194 & e90 \\
\hline
\end{tabular}


Table 1. Cont.

\begin{tabular}{|c|c|c|}
\hline DOI & Previous Page Number & Current Article Number \\
\hline 10.4081/cp.2011.e91 & 195-197 & e91 \\
\hline 10.4081/cp.2011.e92 & 198-199 & e92 \\
\hline 10.4081/cp.2011.e93 & $200-202$ & e93 \\
\hline 10.4081/cp.2011.e94 & $203-204$ & e94 \\
\hline 10.4081/cp.2011.e95 & $205-208$ & e95 \\
\hline 10.4081/cp.2011.e96 & $209-210$ & e96 \\
\hline 10.4081/cp.2011.e97 & $211-212$ & e97 \\
\hline 10.4081/cp.2011.e98 & $213-214$ & e98 \\
\hline 10.4081/cp.2011.e99 & $215-217$ & e99 \\
\hline 10.4081/cp.2011.e100 & $218-221$ & e100 \\
\hline 10.4081/cp.2011.e101 & $222-223$ & e101 \\
\hline 10.4081/cp.2011.e102 & $224-225$ & e102 \\
\hline 10.4081/cp.2011.e103 & $226-227$ & e103 \\
\hline 10.4081/cp.2011.e104 & $228-229$ & e104 \\
\hline 10.4081/cp.2011.e105 & $230-231$ & e105 \\
\hline 10.4081/cp.2011.e106 & $232-233$ & e106 \\
\hline 10.4081/cp.2011.e107 & $234-236$ & e107 \\
\hline 10.4081/cp.2011.e108 & $237-238$ & e108 \\
\hline 10.4081/cp.2011.e109 & 239 & e109 \\
\hline 10.4081/cp.2011.e110 & 240 & e110 \\
\hline 10.4081/cp.2011.e111 & $241-242$ & e111 \\
\hline 10.4081/cp.2011.e112 & $243-244$ & e112 \\
\hline 10.4081/cp.2011.e113 & $245-246$ & e113 \\
\hline 10.4081/cp.2011.e114 & $247-248$ & e114 \\
\hline 10.4081/cp.2011.e115 & $249-250$ & e115 \\
\hline 10.4081/cp.2011.e116 & $251-252$ & e116 \\
\hline 10.4081/cp.2011.e117 & $253-255$ & e117 \\
\hline 10.4081/cp.2011.e118 & $256-258$ & e118 \\
\hline 10.4081/cp.2011.e119 & $259-260$ & e119 \\
\hline 10.4081/cp.2011.e120 & $261-262$ & e120 \\
\hline 10.4081/cp.2011.e121 & $263-264$ & e121 \\
\hline 10.4081/cp.2011.e122 & $265-266$ & e122 \\
\hline 10.4081/cp.2011.e123 & $267-268$ & e123 \\
\hline 10.4081/cp.2011.e124 & $269-273$ & e124 \\
\hline 10.4081/cp.2011.e125 & $274-276$ & e125 \\
\hline 10.4081/cp.2011.e126 & $277-278$ & e126 \\
\hline 10.4081/cp.2011.e127 & $279-281$ & e127 \\
\hline 10.4081/cp.2011.e128 & $282-283$ & e128 \\
\hline 10.4081/cp.2011.e129 & $284-286$ & e129 \\
\hline 10.4081/cp.2011.e130 & $287-289$ & e130 \\
\hline 10.4081/cp.2011.e131 & $290-291$ & e131 \\
\hline 10.4081/cp.2011.e132 & $292-295$ & e132 \\
\hline 10.4081/cp.2011.e133 & $296-298$ & e133 \\
\hline 10.4081/cp.2011.e134 & $299-301$ & e134 \\
\hline 10.4081/cp.2011.e135 & $302-303$ & e135 \\
\hline 10.4081/cp.2011.e136 & $304-305$ & e136 \\
\hline 10.4081/cp.2011.e137 & $306-307$ & e137 \\
\hline 10.4081/cp.2011.e138 & $308-312$ & e138 \\
\hline 10.4081/cp.2011.e139 & $313-314$ & e139 \\
\hline 10.4081/cp.2012.e1 & 1 & e1 \\
\hline 10.4081/cp.2012.e2 & $2-4$ & e2 \\
\hline 10.4081/cp.2012.e3 & $5-6$ & e3 \\
\hline 10.4081/cp.2012.e4 & $7-8$ & e4 \\
\hline 10.4081/cp.2012.e5 & $9-11$ & e5 \\
\hline 10.4081/cp.2012.e6 & $12-13$ & e6 \\
\hline 10.4081/cp.2012.e7 & $14-15$ & e7 \\
\hline 10.4081/cp.2012.e8 & 16 & e8 \\
\hline 10.4081/cp.2012.e9 & $17-18$ & e9 \\
\hline 10.4081/cp.2012.e10 & $19-20$ & e10 \\
\hline
\end{tabular}


Table 1. Cont.

\begin{tabular}{|c|c|c|}
\hline DOI & Previous Page Number & Current Article Number \\
\hline 10.4081/cp.2012.e11 & $21-22$ & e11 \\
\hline 10.4081/cp.2012.e12 & $23-25$ & e12 \\
\hline 10.4081/cp.2012.e13 & $26-27$ & e13 \\
\hline 10.4081/cp.2012.e14 & $28-29$ & e14 \\
\hline 10.4081/cp.2012.e15 & $30-33$ & e15 \\
\hline 10.4081/cp.2012.e16 & $34-36$ & e16 \\
\hline 10.4081/cp.2012.e17 & $37-40$ & e17 \\
\hline 10.4081/cp.2012.e18 & $41-42$ & e18 \\
\hline 10.4081/cp.2012.e19 & $43-44$ & e19 \\
\hline 10.4081/cp.2012.e20 & $45-46$ & e20 \\
\hline 10.4081/cp.2012.e21 & $47-48$ & $\mathrm{e} 21$ \\
\hline 10.4081/cp.2012.e22 & $49-51$ & $\mathrm{e} 22$ \\
\hline 10.4081/cp.2012.e23 & $52-54$ & $\mathrm{e} 23$ \\
\hline 10.4081/cp.2012.e24 & $55-57$ & e24 \\
\hline 10.4081/cp.2012.e25 & $58-59$ & e25 \\
\hline 10.4081/cp.2012.e26 & $60-62$ & e26 \\
\hline 10.4081/cp.2012.e27 & $63-65$ & e27 \\
\hline 10.4081/cp.2012.e28 & $66-68$ & $\mathrm{e} 28$ \\
\hline 10.4081/cp.2012.e29 & $69-70$ & e29 \\
\hline 10.4081/cp.2012.e30 & $71-73$ & e30 \\
\hline 10.4081/cp.2012.e31 & $74-75$ & e31 \\
\hline 10.4081/cp.2012.e32 & $76-77$ & e32 \\
\hline $10.4081 /$ cp.2012.e33 & $78-79$ & e33 \\
\hline 10.4081/cp.2012.e34 & $80-82$ & e34 \\
\hline 10.4081/cp.2012.e35 & $83-84$ & e35 \\
\hline 10.4081/cp.2012.e36 & $85-87$ & e36 \\
\hline 10.4081/cp.2012.e37 & $88-90$ & e37 \\
\hline 10.4081/cp.2012.e38 & $91-92$ & e38 \\
\hline 10.4081/cp.2012.e39 & $94-95$ & e39 \\
\hline 10.4081/cp.2012.e40 & $96-97$ & e40 \\
\hline 10.4081/cp.2012.e41 & 98-99 & e41 \\
\hline $10.4081 /$ cp.2012.e42 & $100-103$ & $\mathrm{e} 42$ \\
\hline 10.4081/cp.2012.e43 & 104 & e43 \\
\hline 10.4081/cp.2012.e44 & $105-106$ & e44 \\
\hline 10.4081/cp.2012.e45 & $108-109$ & $\mathrm{e} 45$ \\
\hline 10.4081/cp.2012.e46 & $108-109$ & e46 \\
\hline 10.4081/cp.2012.e47 & $110-112$ & e47 \\
\hline 10.4081/cp.2012.e48 & $113-115$ & $\mathrm{e} 48$ \\
\hline 10.4081/cp.2012.e49 & $116-117$ & e49 \\
\hline 10.4081/cp.2012.e50 & 118-119 & e50 \\
\hline 10.4081/cp.2012.e51 & $120-122$ & e51 \\
\hline 10.4081/cp.2012.e52 & $123-124$ & e52 \\
\hline $10.4081 /$ cp.2012.e53 & $125-127$ & e53 \\
\hline 10.4081/cp.2012.e54 & $128-129$ & e54 \\
\hline 10.4081/cp.2012.e55 & $130-131$ & e55 \\
\hline 10.4081/cp.2012.e56 & $132-137$ & e56 \\
\hline 10.4081/cp.2012.e57 & $138-140$ & e57 \\
\hline 10.4081/cp.2012.e58 & $141-143$ & e58 \\
\hline 10.4081/cp.2012.e59 & 144-146 & e59 \\
\hline 10.4081/cp.2012.e60 & $147-150$ & e60 \\
\hline 10.4081/cp.2012.e61 & $151-152$ & e61 \\
\hline $10.4081 /$ cp.2012.e62 & $153-154$ & e62 \\
\hline 10.4081/cp.2012.e63 & $155-156$ & e63 \\
\hline 10.4081/cp.2012.e64 & $157-169$ & e64 \\
\hline 10.4081/cp.2012.e65 & $161-163$ & e65 \\
\hline $10.4081 /$ cp.2012.e66 & $164-167$ & e66 \\
\hline 10.4081/cp.2012.e67 & $168-169$ & e67 \\
\hline 10.4081/cp.2012.e68 & $170-172$ & e68 \\
\hline
\end{tabular}


Table 1. Cont.

\begin{tabular}{|c|c|c|}
\hline DOI & Previous Page Number & Current Article Number \\
\hline 10.4081/cp.2012.e69 & $173-174$ & e69 \\
\hline 10.4081/cp.2012.e70 & $175-177$ & e70 \\
\hline 10.4081/cp.2012.e71 & $178-179$ & e71 \\
\hline 10.4081/cp.2012.e72 & $180-181$ & $\mathrm{e} 72$ \\
\hline 10.4081/cp.2012.e73 & $182-183$ & e73 \\
\hline 10.4081/cp.2012.e74 & 184-186 & e74 \\
\hline 10.4081/cp.2012.e75 & $187-188$ & e75 \\
\hline 10.4081/cp.2012.e76 & 189-191 & e76 \\
\hline 10.4081/cp.2012.e77 & $192-193$ & e77 \\
\hline 10.4081/cp.2012.e78 & 194-195 & e78 \\
\hline 10.4081/cp.2012.e79 & $196-198$ & e79 \\
\hline 10.4081/cp.2012.e80 & 199-200 & e80 \\
\hline 10.4081/cp.2012.e81 & $201-202$ & e81 \\
\hline 10.4081/cp.2012.e82 & $203-205$ & e82 \\
\hline 10.4081/cp.2012.e83 & $206-211$ & e83 \\
\hline 10.4081/cp.2012.e84 & $212-215$ & e84 \\
\hline 10.4081/cp.2012.e85 & $216-220$ & e85 \\
\hline $10.4081 /$ cp.2012.e86 & $221-224$ & e86 \\
\hline 10.4081/cp.2012.e87 & $225-227$ & e87 \\
\hline 10.4081/cp.2012.e88 & $228-230$ & e88 \\
\hline 10.4081/cp.2012.e89 & $231-234$ & e89 \\
\hline 10.4081/cp.2013.e1 & $1-2$ & e1 \\
\hline 10.4081/cp.2013.e2 & $3-5$ & e2 \\
\hline 10.4081/cp.2013.e3 & $6-8$ & e3 \\
\hline 10.4081/cp.2013.e4 & $9-10$ & e4 \\
\hline 10.4081/cp.2013.e5 & $11-13$ & e5 \\
\hline 10.4081/cp.2013.e6 & $14-15$ & e6 \\
\hline 10.4081/cp.2013.e7 & $16-17$ & e7 \\
\hline 10.4081/cp.2013.e8 & $18-19$ & e8 \\
\hline 10.4081/cp.2013.e9 & $20-24$ & e9 \\
\hline 10.4081/cp.2013.e10 & $25-26$ & e10 \\
\hline 10.4081/cp.2013.e11 & $27-29$ & e11 \\
\hline 10.4081/cp.2013.e12 & $30-32$ & e12 \\
\hline 10.4081/cp.2013.e13 & $33-34$ & e13 \\
\hline 10.4081/cp.2013.e14 & $35-36$ & e14 \\
\hline 10.4081/cp.2013.e15 & $37-39$ & e15 \\
\hline 10.4081/cp.2013.e16 & $40-42$ & e16 \\
\hline 10.4081/cp.2013.e17 & $43-45$ & e17 \\
\hline 10.4081/cp.2013.e18 & $46-47$ & e18 \\
\hline 10.4081/cp.2013.e19 & $48-50$ & e19 \\
\hline 10.4081/cp.2013.e20 & $51-55$ & e20 \\
\hline 10.4081/cp.2013.e21 & $56-57$ & $\mathrm{e} 21$ \\
\hline 10.4081/cp.2013.e22 & $58-60$ & $\mathrm{e} 22$ \\
\hline 10.4081/cp.2013.e23 & $61-62$ & $\mathrm{e} 23$ \\
\hline 10.4081/cp.2013.e24 & $63-64$ & $\mathrm{e} 24$ \\
\hline 10.4081/cp.2013.e25 & $65-67$ & $\mathrm{e} 25$ \\
\hline 10.4081/cp.2013.e26 & $68-70$ & $\mathrm{e} 26$ \\
\hline 10.4081/cp.2013.e27 & $71-73$ & $\mathrm{e} 27$ \\
\hline 10.4081/cp.2013.e28 & $74-76$ & $\mathrm{e} 28$ \\
\hline 10.4081/cp.2013.e29 & $77-83$ & e29 \\
\hline 10.4081/cp.2013.e30 & $84-86$ & e30 \\
\hline 10.4081/cp.2013.e31 & $87-90$ & e31 \\
\hline 10.4081/cp.2013.e32 & $91-92$ & e32 \\
\hline $10.4081 /$ cp.2014.599 & $34-37$ & 599 \\
\hline $10.4081 /$ cp.2014.604 & $1-3$ & 604 \\
\hline $10.4081 /$ cp.2014.605 & $12-14$ & 605 \\
\hline 10.4081/cp.2014.608 & $4-6$ & 608 \\
\hline 10.4081/cp.2014.609 & $10-11$ & 609 \\
\hline $10.4081 /$ cp.2014.610 & $7-9$ & 610 \\
\hline
\end{tabular}


Table 1. Cont.

\begin{tabular}{|c|c|c|}
\hline DOI & Previous Page Number & Current Article Number \\
\hline $10.4081 /$ cp. 2014.628 & $17-21$ & 628 \\
\hline $10.4081 /$ cp.2014.632 & $15-16$ & 632 \\
\hline $10.4081 /$ cp. 2014.635 & $24-26$ & 635 \\
\hline $10.4081 /$ cp. 2014.637 & $22-23$ & 637 \\
\hline $10.4081 /$ cp.2014.642 & $50-52$ & 642 \\
\hline 10.4081/cp.2014.644 & 46 & 644 \\
\hline $10.4081 /$ cp.2014.646 & $43-45$ & 646 \\
\hline $10.4081 /$ cp.2014.647 & $55-57$ & 647 \\
\hline $10.4081 /$ cp.2014.648 & $58-59$ & 648 \\
\hline $10.4081 /$ cp.2014.651 & $30-33$ & 651 \\
\hline $10.4081 /$ cp.2014.653 & $27-29$ & 653 \\
\hline $10.4081 /$ cp.2014.656 & $38-39$ & 656 \\
\hline 10.4081/cp.2014.659 & $47-49$ & 659 \\
\hline $10.4081 /$ cp.2014.660 & $64-65$ & 660 \\
\hline 10.4081/cp.2014.661 & $53-54$ & 661 \\
\hline $10.4081 /$ cp.2014.664 & $40-42$ & 664 \\
\hline $10.4081 /$ cp. 2014.670 & $66-69$ & 670 \\
\hline $10.4081 /$ cp.2014.679 & $74-76$ & 679 \\
\hline $10.4081 /$ cp.2014.686 & $60-63$ & 686 \\
\hline $10.4081 /$ cp.2014.725 & 70 & 725 \\
\hline $10.4081 /$ cp. 2014.726 & 71 & 726 \\
\hline $10.4081 /$ cp. 2014.738 & 72 & 738 \\
\hline $10.4081 /$ cp.2014.739 & 73 & 739 \\
\hline $10.4081 /$ cp.2015.668 & $3-5$ & 668 \\
\hline $10.4081 /$ cp.2015.688 & $1-2$ & 688 \\
\hline $10.4081 /$ cp.2015.697 & $6-7$ & 697 \\
\hline $10.4081 /$ cp.2015.699 & $11-13$ & 699 \\
\hline $10.4081 /$ cp.2015.701 & 17-19 & 701 \\
\hline 10.4081/cp.2015.706 & $8-10$ & 706 \\
\hline $10.4081 /$ cp.2015.707 & $37-39$ & 707 \\
\hline $10.4081 /$ cp.2015.708 & $26-27$ & 708 \\
\hline $10.4081 /$ cp. 2015.717 & $28-31$ & 717 \\
\hline $10.4081 /$ cp.2015.722 & $14-16$ & 722 \\
\hline $10.4081 /$ cp.2015.727 & $32-34$ & 727 \\
\hline $10.4081 /$ cp.2015.733 & $23-25$ & 733 \\
\hline $10.4081 /$ cp. 2015.734 & $40-43$ & 734 \\
\hline $10.4081 /$ cp.2015.736 & $53-55$ & 736 \\
\hline $10.4081 /$ cp.2015.740 & $60-62$ & 740 \\
\hline $10.4081 /$ cp.2015.741 & $50-52$ & 741 \\
\hline $10.4081 /$ cp.2015.746 & $56-57$ & 746 \\
\hline $10.4081 /$ cp. 2015.748 & $20-22$ & 748 \\
\hline 10.4081/cp.2015.749 & $47-49$ & 749 \\
\hline 10.4081/cp.2015.752 & $44-46$ & 752 \\
\hline $10.4081 /$ ср. 2015.754 & $35-36$ & 754 \\
\hline $10.4081 /$ cp.2015.756 & $58-59$ & 756 \\
\hline $10.4081 /$ cp.2015.763 & $84-87$ & 763 \\
\hline $10.4081 /$ cp.2015.767 & $72-74$ & 767 \\
\hline $10.4081 /$ cp.2015.768 & $78-80$ & 768 \\
\hline $10.4081 /$ cp. 2015.775 & $75-77$ & 775 \\
\hline $10.4081 /$ cp. 2015.776 & 104-106 & 776 \\
\hline $10.4081 /$ cp. 2015.777 & $94-98$ & 777 \\
\hline $10.4081 /$ cp.2015.780 & $68-71$ & 780 \\
\hline $10.4081 /$ cp. 2015.781 & $65-67$ & 781 \\
\hline $10.4081 /$ cp. 2015.784 & $88-90$ & 784 \\
\hline $10.4081 /$ cp. 2015.788 & $63-64$ & 788 \\
\hline 10.4081/cp.2015.792 & 91-93 & 792 \\
\hline $10.4081 /$ cp.2015.793 & $124-127$ & 793 \\
\hline $10.4081 /$ cp.2015.804 & $116-118$ & 804 \\
\hline $10.4081 /$ cp.2015.810 & $113-115$ & 810 \\
\hline
\end{tabular}


Table 1. Cont.

\begin{tabular}{|c|c|c|}
\hline DOI & Previous Page Number & Current Article Number \\
\hline 10.4081/cp.2015.814 & 99-103 & 814 \\
\hline $10.4081 /$ cp.2015.818 & $122-123$ & 818 \\
\hline $10.4081 /$ cp.2015.819 & $119-120$ & 819 \\
\hline $10.4081 /$ cp. 2015.821 & 107-109 & 821 \\
\hline $10.4081 /$ cp. 2016.786 & $53-54$ & 786 \\
\hline 10.4081/cp.2016.811 & $1-2$ & 811 \\
\hline $10.4081 /$ cp.2016.813 & $13-15$ & 813 \\
\hline $10.4081 /$ cp.2016.816 & $21-23$ & 816 \\
\hline $10.4081 /$ cp.2016.820 & $3-5$ & 820 \\
\hline $10.4081 /$ cp.2016.822 & $6-7$ & 822 \\
\hline $10.4081 /$ cp.2016.823 & $11-12$ & 823 \\
\hline $10.4081 /$ cp.2016.824 & $42-45$ & 824 \\
\hline $10.4081 /$ cp. 2016.828 & $16-17$ & 828 \\
\hline $10.4081 /$ cp.2016.832 & $40-41$ & 832 \\
\hline 10.4081/cp.2016.837 & $46-48$ & 837 \\
\hline $10.4081 /$ cp. 2016.838 & $18-20$ & 838 \\
\hline $10.4081 /$ cp. 2016.840 & $8-10$ & 840 \\
\hline $10.4081 /$ cp.2016.841 & $71-72$ & 841 \\
\hline $10.4081 /$ cp. 2016.843 & $27-30$ & 843 \\
\hline 10.4081/cp.2016.844 & $49-50$ & 844 \\
\hline $10.4081 /$ cp. 2016.846 & $51-52$ & 846 \\
\hline $10.4081 /$ cp.2016.847 & $24-26$ & 847 \\
\hline $10.4081 /$ cp. 2016.848 & $68-70$ & 848 \\
\hline $10.4081 /$ cp.2016.849 & $96-97$ & 849 \\
\hline 10.4081/cp.2016.852 & $55-56$ & 852 \\
\hline $10.4081 /$ cp.2016.853 & $113-116$ & 853 \\
\hline $10.4081 /$ cp.2016.855 & $35-41$ & 855 \\
\hline $10.4081 /$ cp.2016.856 & $38-39$ & 856 \\
\hline 10.4081/cp.2016.862 & $76-77$ & 862 \\
\hline $10.4081 /$ cp.2016.865 & 98-101 & 865 \\
\hline $10.4081 /$ cp.2016.866 & $81-85$ & 866 \\
\hline $10.4081 /$ cp. 2016.868 & $65-67$ & 868 \\
\hline $10.4081 /$ cp.2016.871 & $61-64$ & 871 \\
\hline $10.4081 /$ cp.2016.873 & $57-60$ & 873 \\
\hline $10.4081 /$ cp. 2016.878 & $78-80$ & 878 \\
\hline $10.4081 /$ cp.2016.879 & $108-112$ & 879 \\
\hline $10.4081 /$ cp.2016.881 & $73-75$ & 881 \\
\hline 10.4081/cp.2016.884 & 93-95 & 884 \\
\hline $10.4081 /$ cp.2016.885 & 117-118 & 885 \\
\hline $10.4081 /$ cp.2016.887 & 89-92 & 887 \\
\hline $10.4081 /$ cp.2016.893 & $105-107$ & 893 \\
\hline $10.4081 /$ cp.2016.895 & $86-88$ & 895 \\
\hline 10.4081/cp.2016.897 & $122-125$ & 897 \\
\hline $10.4081 /$ cp.2016.903 & $119-121$ & 903 \\
\hline $10.4081 /$ cp.2017.857 & $48-50$ & 857 \\
\hline $10.4081 /$ cp. 2017.888 & $1-6$ & 888 \\
\hline 10.4081/cp.2017.890 & $7-10$ & 890 \\
\hline $10.4081 /$ cp.2017.898 & $23-27$ & 898 \\
\hline $10.4081 /$ cp.2017.904 & $31-33$ & 904 \\
\hline $10.4081 /$ cp. 2017.911 & $15-19$ & 911 \\
\hline $10.4081 /$ cp. 2017.912 & $11-14$ & 912 \\
\hline $10.4081 /$ cp.2017.918 & $61-63$ & 918 \\
\hline $10.4081 /$ cp. 2017.920 & $39-40$ & 920 \\
\hline $10.4081 /$ cp. 2017.921 & $20-22$ & 921 \\
\hline $10.4081 /$ cp.2017.922 & $64-66$ & 922 \\
\hline $10.4081 /$ cp. 2017.928 & $28-30$ & 928 \\
\hline $10.4081 /$ cp.2017.930 & $54-55$ & 930 \\
\hline 10.4081 /cp.2017.932 & $36-38$ & 932 \\
\hline $10.4081 /$ cp.2017.933 & $41-44$ & 933 \\
\hline
\end{tabular}


Table 1. Cont.

\begin{tabular}{|c|c|c|}
\hline DOI & Previous Page Number & Current Article Number \\
\hline 10.4081/cp.2017.936 & $56-59$ & 936 \\
\hline 10.4081/cp.2017.938 & $45-47$ & 938 \\
\hline 10.4081/cp.2017.939 & 99-100 & 939 \\
\hline $10.4081 /$ cp.2017.942 & $84-87$ & 942 \\
\hline $10.4081 /$ cp.2017.943 & $66-69$ & 943 \\
\hline 10.4081/cp.2017.946 & $70-71$ & 946 \\
\hline $10.4081 /$ cp.2017.950 & $78-80$ & 950 \\
\hline $10.4081 /$ cp.2017.951 & $75-77$ & 951 \\
\hline 10.4081/cp.2017.952 & $51-53$ & 952 \\
\hline $10.4081 /$ cp.2017.953 & $72-74$ & 953 \\
\hline 10.4081/cp.2017.955 & 96-98 & 955 \\
\hline $10.4081 /$ cp.2017.957 & $101-103$ & 957 \\
\hline $10.4081 /$ cp.2017.958 & $81-83$ & 958 \\
\hline $10.4081 /$ cp.2017.960 & $88-91$ & 960 \\
\hline 10.4081/cp.2017.961 & $158-159$ & 961 \\
\hline $10.4081 /$ cp.2017.969 & 104-106 & 969 \\
\hline $10.4081 /$ cp.2017.973 & $124-127$ & 973 \\
\hline $10.4081 /$ cp.2017.975 & $107-110$ & 975 \\
\hline $10.4081 /$ cp.2017.976 & $93-95$ & 976 \\
\hline $10.4081 /$ cp.2017.977 & $117-119$ & 977 \\
\hline $10.4081 /$ cp.2017.979 & $111-113$ & 979 \\
\hline $10.4081 /$ cp.2017.982 & $155-157$ & 982 \\
\hline $10.4081 /$ cp.2017.985 & $148-149$ & 985 \\
\hline 10.4081/cp.2017.987 & $131-136$ & 987 \\
\hline 10.4081/cp.2017.992 & $141-144$ & 992 \\
\hline $10.4081 /$ cp.2017.999 & $137-140$ & 999 \\
\hline 10.4081/cp.2017.1002 & $121-123$ & 1002 \\
\hline $10.4081 /$ cp.2017.1006 & $160-162$ & 1006 \\
\hline 10.4081/cp.2017.1008 & $150-154$ & 1008 \\
\hline 10.4081/cp.2017.1009 & $145-147$ & 1009 \\
\hline $10.4081 /$ cp.2017.1018 & $163-165$ & 1018 \\
\hline $10.4081 /$ ср.2018.981 & $1-4$ & 981 \\
\hline 10.4081/cp.2018.991 & $28-30$ & 991 \\
\hline 10.4081/cp.2018.1004 & $12-14$ & 1004 \\
\hline $10.4081 /$ cp.2018.1007 & $5-7$ & 1007 \\
\hline $10.4081 /$ cp.2018.1021 & $25-27$ & 1021 \\
\hline $10.4081 /$ cp.2018.1023 & $8-11$ & 1023 \\
\hline 10.4081/cp.2018.1031 & $15-18$ & 1031 \\
\hline 10.4081/cp.2018.1035 & $80-81$ & 1035 \\
\hline $10.4081 /$ cp.2018.1037 & $45-46$ & 1037 \\
\hline $10.4081 /$ cp.2018.1038 & $49-51$ & 1038 \\
\hline $10.4081 /$ cp.2018.1039 & $47-48$ & 1039 \\
\hline 10.4081/cp.2018.1040 & $38-41$ & 1040 \\
\hline $10.4081 /$ cp.2018.1044 & $19-20$ & 1044 \\
\hline $10.4081 /$ cp.2018.1046 & $36-37$ & 1046 \\
\hline $10.4081 /$ cp.2018.1047 & $31-35$ & 1047 \\
\hline 10.4081/cp.2018.1053 & $42-44$ & 1053 \\
\hline 10.4081/cp.2018.1054 & $59-62$ & 1054 \\
\hline 10.4081/cp.2018.1055 & $21-24$ & 1055 \\
\hline $10.4081 /$ cp.2018.1057 & $67-68$ & 1057 \\
\hline 10.4081/cp.2018.1061 & $63-66$ & 1061 \\
\hline 10.4081/cp.2018.1065 & 99-101 & 1065 \\
\hline $10.4081 /$ cp.2018.1069 & $52-54$ & 1069 \\
\hline $10.4081 /$ cp.2018.1070 & $85-87$ & 1070 \\
\hline $10.4081 /$ cp.2018.1071 & $91-93$ & 1071 \\
\hline 10.4081/cp.2018.1072 & $55-58$ & 1072 \\
\hline 10.4081/cp.2018.1073 & $82-84$ & 1073 \\
\hline 10.4081/cp.2018.1079 & $95-98$ & 1079 \\
\hline $10.4081 /$ cp.2018.1085 & $69-72$ & 1085 \\
\hline
\end{tabular}


Table 1. Cont.

\begin{tabular}{|c|c|c|}
\hline DOI & Previous Page Number & Current Article Number \\
\hline 10.4081/cp.2018.1089 & $73-79$ & 1089 \\
\hline $10.4081 /$ cp.2018.1093 & $88-90$ & 1093 \\
\hline $10.4081 /$ cp.2018.1094 & $102-103$ & 1094 \\
\hline $10.4081 /$ cp.2018.1095 & $111-113$ & 1095 \\
\hline $10.4081 /$ cp.2018.1097 & $109-110$ & 1097 \\
\hline $10.4081 /$ cp.2018.1102 & $106-108$ & 1102 \\
\hline $10.4081 /$ cp.2018.1104 & 104-105 & 1104 \\
\hline 10.4081/cp.2019.1086 & $1-3$ & 1086 \\
\hline $10.4081 /$ cp.2019.1091 & $9-11$ & 1091 \\
\hline $10.4081 /$ cp.2019.1096 & $4-5$ & 1096 \\
\hline $10.4081 /$ cp.2019.1110 & $23-25$ & 1110 \\
\hline $10.4081 /$ cp.2019.1111 & $87-89$ & 1111 \\
\hline $10.4081 /$ cp.2019.1112 & $51-54$ & 1112 \\
\hline $10.4081 /$ cp.2019.1114 & $6-8$ & 1114 \\
\hline $10.4081 /$ cp.2019.1117 & $55-57$ & 1117 \\
\hline $10.4081 /$ cp.2019.1119 & $32-37$ & 1119 \\
\hline $10.4081 /$ cp.2019.1124 & $45-47$ & 1124 \\
\hline $10.4081 /$ cp.2019.1125 & $38-41$ & 1125 \\
\hline $10.4081 /$ cp.2019.1126 & $18-22$ & 1126 \\
\hline $10.4081 /$ cp.2019.1128 & $26-28$ & 1128 \\
\hline $10.4081 /$ cp.2019.1129 & $29-31$ & 1129 \\
\hline $10.4081 /$ cp.2019.1132 & $12-17$ & 1132 \\
\hline $10.4081 /$ cp.2019.1134 & $65-68$ & 1134 \\
\hline $10.4081 /$ cp.2019.1135 & $81-83$ & 1135 \\
\hline $10.4081 /$ cp.2019.1140 & $90-95$ & 1140 \\
\hline $10.4081 /$ cp.2019.1141 & $73-74$ & 1141 \\
\hline $10.4081 /$ cp.2019.1142 & $75-77$ & 1142 \\
\hline $10.4081 /$ cp.2019.1143 & $111-113$ & 1143 \\
\hline $10.4081 /$ cp.2019.1144 & $61-64$ & 1144 \\
\hline $10.4081 /$ cp.2019.1146 & $48-50$ & 1146 \\
\hline $10.4081 /$ cp.2019.1153 & $42-44$ & 1153 \\
\hline $10.4081 /$ cp.2019.1157 & $58-60$ & 1157 \\
\hline $10.4081 /$ cp.2019.1161 & 96-101 & 1161 \\
\hline $10.4081 /$ cp.2019.1172 & $78-80$ & 1172 \\
\hline 10.4081/cp.2019.1176 & $102-103$ & 1176 \\
\hline $10.4081 /$ cp.2019.1177 & $84-86$ & 1177 \\
\hline $10.4081 /$ cp.2019.1179 & $69-72$ & 1179 \\
\hline $10.4081 /$ cp.2019.1184 & $127-132$ & 1184 \\
\hline $10.4081 /$ cp.2019.1187 & $114-117$ & 1187 \\
\hline $10.4081 /$ cp.2019.1188 & $106-108$ & 1188 \\
\hline $10.4081 /$ cp.2019.1190 & $109-110$ & 1190 \\
\hline $10.4081 /$ cp.2019.1191 & $118-122$ & 1191 \\
\hline $10.4081 /$ cp.2019.1209 & $123-126$ & 1209 \\
\hline $10.4081 /$ cp.2020.1200 & $4-7$ & 1200 \\
\hline $10.4081 /$ cp.2020.1204 & $53-55$ & 1204 \\
\hline 10.4081/cp.2020.1205 & $42-46$ & 1205 \\
\hline $10.4081 /$ cp.2020.1211 & $74-77$ & 1211 \\
\hline $10.4081 /$ cp.2020.1214 & $34-36$ & 1214 \\
\hline 10.4081/cp.2020.1216 & $1-3$ & 1216 \\
\hline $10.4081 /$ cp.2020.1217 & $56-58$ & 1217 \\
\hline $10.4081 /$ cp.2020.1218 & $8-11$ & 1218 \\
\hline $10.4081 /$ cp.2020.1221 & $12-14$ & 1221 \\
\hline $10.4081 /$ cp.2020.1222 & $15-16$ & 1222 \\
\hline $10.4081 /$ cp.2020.1226 & $86-88$ & 1226 \\
\hline $10.4081 /$ cp.2020.1234 & $17-19$ & 1234 \\
\hline $10.4081 /$ cp.2020.1242 & $82-85$ & 1242 \\
\hline $10.4081 /$ cp.2020.1248 & $49-52$ & 1248 \\
\hline 10.4081/cp.2020.1249 & $37-39$ & 1249 \\
\hline $10.4081 /$ cp.2020.1251 & $20-23$ & 1251 \\
\hline
\end{tabular}


Table 1. Cont.

\begin{tabular}{ccc}
\hline DOI & Previous Page Number & Current Article Number \\
\hline 10.4081 /cp.2020.1253 & $89-92$ & 1253 \\
$10.4081 /$ cp.2020.1254 & $40-41$ & 1254 \\
10.4081 /cp.2020.1255 & $31-33$ & 1255 \\
$10.4081 /$ cp.2020.1257 & $66-69$ & 1257 \\
$10.4081 /$ cp.2020.1258 & $70-73$ & 1258 \\
$10.4081 /$ cp.2020.1265 & $59-61$ & 1265 \\
$10.4081 /$ cp.2020.1267 & $47-48$ & 1267 \\
$10.4081 /$ cp.2020.1270 & $100-102$ & 1270 \\
$10.4081 /$ cp.2020.1271 & $24-30$ & 1271 \\
$10.4081 /$ cp.2020.1276 & $93-95$ & 1276 \\
$10.4081 /$ cp.2020.1281 & $62-65$ & 1281 \\
$10.4081 /$ cp.2020.1285 & $78-81$ & 1285 \\
$10.4081 /$ cp.2020.1292 & $96-99$ & 1292 \\
\hline
\end{tabular}

Conflicts of Interest: The author declares no conflict of interest.

\section{Reference}

1. Clinics and Practice Homepage. Available online: https://www.mdpi.com/journal/clinpract (accessed on 10 February 2022). 